\title{
Multi-resolution Image Fusion Algorithm Based on Improved Regional Cross Entropy and Morphology
}

\author{
Wen $\mathrm{Ge}^{\mathrm{a}}$, Jin $\mathrm{Li} \mathrm{Xu}^{\mathrm{b}}$ and Peng $\mathrm{Li}^{\mathrm{c}}$ \\ School of Electronics and Information Engineering, Shenyang Aerospace University, No.37 Daoyi \\ South Avenue, Daoyi Development District, 110136 Shenyang, China \\ agewenbox72@sina.com.cn, ${ }^{\mathrm{a} x u j i n l i 73 @ 163 . c o m, ~}{ }^{\mathrm{c}} 31731830 @ q q . c o m$
}

Keywords: Regional cross entropy; Wavelet transform; Image fusion; Morphology; Edge information

\begin{abstract}
Based on image contents, the better to simulate the process pattern of human eyes vision, an image fusion method that emphasizing edge preserving is proposed. Through wavelet transform, an improved regional cross entropy fusion rule is used for the low-frequency component which reflects approximate contents, the fusion method for incorporation of the maximum morphology edge measuring and weighted variance analysis is used for the high-frequency component which reflects detail features of image. Finally, the fusion image is reconstructed through an inverse transform of wavelet. Experimental results show that by using this algorithm, the mutual information between the images can be fused organically, the image clarity is raised, the details of fusion image are enhanced, and the edge information are reappeared better. Strong support for the follow-up information analysis and extractive ability of the images are provided.
\end{abstract}

\section{Introduction}

Image fusion is the process to comprehend organically the complementary information and redundant data from several sensors images to obtain the image with richer details, more reliable and easier-to-understand and read for the same scene. There are many image fusion methods, among others, the wavelet transform is fully used for image fusion[1-6] because of its good time frequency localization property and multi-scale analytical ability. The multi-resolution wavelet transform is adopted. The wavelet coefficient and scale coefficient on pixel level are analyzed, treated and fused. The fusion rule based on improved region cross entropy is proposed for the low-frequency component, this method is by means of judging the similarity of being fused images local region, and adopts different fusion strategy to fuse. It can reflect the approximate contents of the image better. The fusion rule based on maximum morphology edge detection and weighted variance is proposed for the high-frequency component. It obtains the fusion effect with better visual property and richer and obvious details, especially for the edge information.

\section{Wavelet Decomposition of Image}

Because the wavelet transform is possessed of the good localization property both in frequency domain and time domain simultaneously, it is used widely in image treatment and analyses. Mallat proposed the algorithm of wavelet transform rapid-decomposition and reconstruction, using two one-dimension filters realized the wavelet rapid-decomposition for the two-dimension image, and then using two one-dimension reconstruction filters realized the image reconstruction. Mallat algorithm decomposition equation under the scale $j$ is [7]: 


$$
\left\{\begin{array}{l}
C_{j}=H_{r} H_{c} C_{j-1} \\
D_{j}^{1}=H_{r} G_{c} C_{j-1} \\
D_{j}^{2}=G_{r} H_{c} C_{j-1} \\
D_{j}^{3}=G_{r} G_{c} C_{j-1}
\end{array} \quad(j=0,-1, \ldots,-J) .\right.
$$

Where, $H$ and $G$ is one-dimension mirror filter operator respectively, the lower mark $r$ and $c$ represents the line operation and column operation for matrix respectively, and the corresponding two-dimension image reconstruction algorithm is:

$$
C_{j-1}=H_{r}^{*} H_{c}^{*} C_{j}+H_{r}^{*} G_{c}^{*} D_{j}^{1}+G_{r}^{*} H_{c}^{*} D_{j}^{2}+G_{r}^{*} G_{c}^{*} D_{j}^{3} \quad(j=-J,-J+1, \ldots,-1) .
$$

Where $H^{*}$ and $G^{*}$ is the conjugate transposed matrix of the $H$ and $G$ respectively; and $C_{j}$ is the approximation of the source image on $2^{-j}$ resolving power (image low-frequency part); $D_{j}^{\varepsilon}(\varepsilon=1,2,3)$ is the error of the approximation (image high-frequency part).

\section{Edge Detection Based on Mathematical Morphology}

Mathematical morphology is an emerging image discipline which established on the set theory foundation. Edge detection based on mathematical morphology method, does not look like differentiate to be sensitive to the noise, extracting edge is smoother, image skeleton is continual, reflects the image characteristic information effectively. Below gives 4 fundamental operation definitions in mathematical morphology.

Corrosion (or erosion): $\boldsymbol{A} \Theta \boldsymbol{B}=\left\{x \mid(\boldsymbol{B})_{x} \subseteq \boldsymbol{A}\right\}$.

Inflation (or expansion): $\left.\boldsymbol{A} \oplus \boldsymbol{B}=\left\{x \mid(\hat{\boldsymbol{B}})_{x} \cap \boldsymbol{A} \neq \boldsymbol{\phi}\right]\right\}$.

Open: $\boldsymbol{A} \circ \boldsymbol{B}=(\boldsymbol{A \Theta B}) \oplus \boldsymbol{B}$.

Close: $\boldsymbol{A} \bullet \boldsymbol{B}=(\boldsymbol{A} \oplus \boldsymbol{B}) \Theta \boldsymbol{B}$.

Where, $\boldsymbol{A}$ is the image set, $\boldsymbol{B}$ is the structural element, $\hat{\boldsymbol{B}}$ expresses the $\boldsymbol{B}$ reflection. In these 4 kind of fundamental operations, the corrosion and the inflation is mutually antithesis operation, open and close is mutually antithesis operation. The corrosion is a process in which an object reduces along the boundary; the inflation is a process in which all background spots contacted with the aimed object merge into the object. Open operation has the function to delete tiny object, in slender place to separate object and to smooth big object boundary; close operation has the function to fill material in vivo tiny cavity and to connect the neighbor goal.

Using open operation characteristic, carries on the edge detection to the high frequency sub-image, eliminates edge other unessential content. In the edge detection process, the selection of structural element directly influences image edge extraction effect. Choosing the appropriate structural element is very important. The structural element is in fact a gradation "shape" inside a small window, i.e. when window size is certain, only needs to determine each point value in the window [8]. The general window size adopts $3 \times 3,5 \times 5,7 \times 7$, in which $3 \times 3$ window speed is quickest, edge extraction is finest. Therefore the $3 \times 3$ plane structural element is chosen.

\section{Image Fusion Rule}

In the image fusion process, designing the reasonable image fusion rule is the key to obtain the high quality fusion image. The fusion rule is proposed, which maintains and restores the source images detail characteristic while raises the image clarity. The high frequency information and low frequency information fusion process are described in detail below. 
Low-frequency Information Fusion. Low-frequency information is the approximate description of source image. It includes the average gradation and texture information etc. of source image, meanwhile, according to the criterion of "human visual system property theory"(HVS)[9], human eyes are more sensitive for the low resolution band of image and less sensitive for the high one. From the criterion, we know that the low frequency information is possessed of great effect on resuming the image quality. So a fusion rule based on improved regional cross entropy is proposed for low-frequency component. The cross entropy is also called relative entropy, it can be used for measuring the difference between the two images. This is the relative measurement of the containing information of the two images. Cross entropy is:

$$
C(p, q)=\sum_{i=1}^{M} p_{i} \ln \left(p_{i} / q_{i}\right) .
$$

Where, $p_{i}$ is the radio between pixel number with the gradation equal to $i$ and the total pixel of the image. $p_{i} / q_{i}$ in the cross entropy defined equation may be greater than 1 , or may be less than 1 . This makes $\ln \left(p_{i} / q_{i}\right)$ may be positive or may be negative, so there will be positive and negative mutual offset. Therefore, cross entropy defined by Eq. 7 can not reflect the difference between the two images correctly. To avoid this condition be happened, an improved cross entropy[10] is adopted.

$$
C^{\prime}(p, q)=\sum_{i=1}^{M} p_{i}\left|\ln \left(p_{i} / q_{i}\right)\right|
$$

Because the local property of image is expressed and reflected by many pixels in the region, the adopting region cross entropy reflects the similarity of the two being fused image local region. The region window selects window $3 * 3$, the regional cross entropy of two being fused images is:

$$
C^{\prime *}(p, q)=\sum_{i=1}^{M} \sum_{j=1}^{N} p_{i j}\left|\ln \left(p_{i j} / q_{i j}\right)\right| .
$$

Where: $p_{i j}$ is the regional gradation distribution in image $\mathrm{A} ; q_{i j}$ is the regional gradation distribution in image $\mathrm{B}$.

$$
P_{i j}=B(i, j) / \sum_{i=1}^{M} \sum_{j=1}^{N} B(i, j) .
$$

Where: $B(i, j)$ is the gradation value of the spot $(i, j)$ in the regional image.

While the difference between the two images is littler, the littler the cross entropy is; while the difference between the two images is larger, the larger the cross entropy is. Supposed, threshold is $T$.

While $C^{\prime *}(p, q)<T$, the fusion algorithm for low-frequency is:

$$
L_{F}=\frac{E_{f A}}{E_{f A}+E_{f B}} L_{A}+\frac{E_{f B}}{E_{f A}+E_{f B}} L_{B} .
$$

Where: $L_{A}, L_{B}$ and $L_{F}$ is the low-frequency coefficient of the two being fused images and the after-fused image respectively. $E_{f A}, E_{f B}$ is the regional information entropy of image A and image B respectively. According to the principle of Shannon's information entropy theory, the regional information entropy $E_{f}$ is defined:

$$
E_{f}=-\sum_{i=1}^{M} \sum_{j=1}^{N} P_{i j} \log _{2} P_{i j} .
$$

While $C^{*}(p, q) \geq T$, the fusion algorithm is: 


$$
L_{F}=\left\{\begin{array}{ll}
L_{A} & E_{f A} \geq E_{f B} \\
L_{B} & E_{f A}<E_{f B}
\end{array} .\right.
$$

Through the above fusion rule, the information supplied by low-frequency component can be fused together effectively.

High-frequency Information Fusion. High-frequency information contains important property and detail information of source image. The key of fusion is to extract the information from the source image effectively, and to realize effective demonstration in the final fusion image. A high-frequency fusion rule based on edge protection is designed, that is, the fusion method for incorporation of the maximum morphology edge detection and weighted variance analysis constructs the high-frequency component of image.

Before the fusion of the high-frequency component, the edge points in the high-frequency component are detected by using mathematical morphology open operation. The data fusion for the non-edge points in the being fused image is performed by using weighted variance analysis method. Variance reflects the dispersion ratio of the image gradation relative to the average gradation. In some degree, variance also can be used for evaluation the degree of image contrast.

$$
\sigma=\sqrt{\sum_{i=1}^{M} \sum_{j=1}^{N}(z(i, j)-\bar{z})^{2} /(M \times N)} .
$$

Where, $z(i, j)$ is the gradation of non-edge points, $\bar{z}$ is the average gradation of all points in the being fused image. The high-frequency information fusion of the non-edge points in the source image is performed by using Eq. 15 .

$$
D_{F}^{H}\left(x_{i}, y_{j}\right)=\frac{\sigma_{A}}{\sigma_{A}+\sigma_{B}} D_{A}+\frac{\sigma_{B}}{\sigma_{A}+\sigma_{B}} D_{B} .
$$

To sum up, after the maximum morphology edge detection and weighted variance analysis for the source image A and B are performed, the high-frequency component is obtained.

$$
D_{F}(i, j)= \begin{cases}\max \left(D_{A}(i, j), D_{B}(i, j)\right) & h=h_{\max }=h_{\max 2} \\ D_{A}(i, j) & h=h_{\max }, h \neq h_{\max 2} \\ D_{B}(i, j) & h \neq h_{\max }, h=h_{\max 2} \\ D_{F}^{H}(i, j) & h \neq h_{\max 1}, h \neq h_{\max 2}\end{cases}
$$

From Eq. 16 we know, at first, the edge points and non-edge points in the source image are determined by using Eq. 5. If point $(i, j)$ is the same edge point of source image $\mathrm{A}$ and $\mathrm{B}$, takes the large wavelet coefficient for the high-frequency component of fusion image; if point $(i, j)$ is the edge point of source image $\mathrm{A}$ or $\mathrm{B}$, takes the corresponding wavelet coefficient for the high-frequency component of fusion image; if point $(i, j)$ is not the edge point of source image $\mathrm{A}$ and $\mathrm{B}$, takes the wavelet coefficient calculated by Eq. 15 for the high-frequency component of fusion image.

Specific Steps of the Fusion Algorithm. According to the analysis above, the basic steps by using wavelet transform to perform image fusion are following:

(1)For the being fused source image A and B, wavelet transform decomposition are performed respectively;

(2) The corresponding low-frequency information of source images A and B are merged by using Eq. 13 ;

(3) The edge points and non-edge points of high-frequency component in the source image A and B are determined by using Eq. 5. The high-frequency component corresponding to the edge points is protected; the high-frequency fusion for the non-edge points is performed. Then the high-frequency component of fusion image is obtained by using Eq. 16. 
(4)The inverse wavelet transform for the high-frequency information and low-frequency information of the fusion image are performed, information of the fusion image is performed, the reconstructed image is obtained.

\section{Experiment Results and Evaluation}

In order to verify the effectiveness of the proposed algorithm, a group of fusion experiments for CT image and MRI image from Fig. 1 are performed by comparison with wavelet transform and Laplacian pyramid. Fig.1(a), (b) is CT, MRI respectively; Fig.1(c) is Laplacian pyramid; Fig.1(d) is wavelet transform algorithm; Fig.1(e) is the proposed algorithm. This fusion method of Laplacian pyramid and wavelet transform, adopts the incorporation of the maximum local modulus and weighted variance analysis for the high-frequency component, and adopts cross entropy weighted fusion rule for the low-frequency component. To view from visual effect of fusion results, the fusion effect of Fig. 1(e) is the best, it remains the detail characteristic information of image better, the target is clear, and improves the visual effect obviously; the fusion effect of Fig. 1(d) and Fig. 1(c) are relatively poor, the edge is discontinuous, and the contract is lost so much.

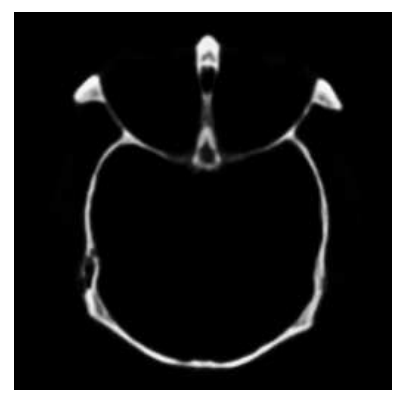

(a) CT image

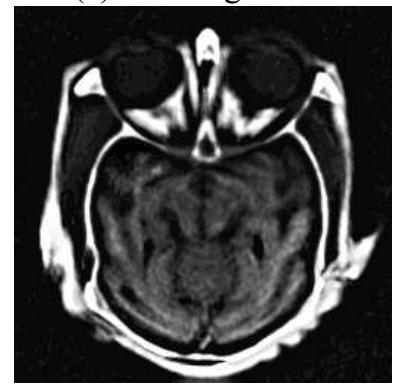

(e) Proposed algorithm

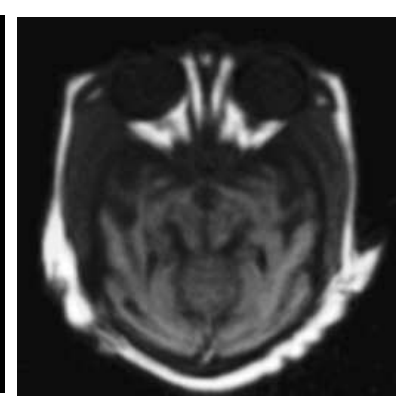

(b) MRI image

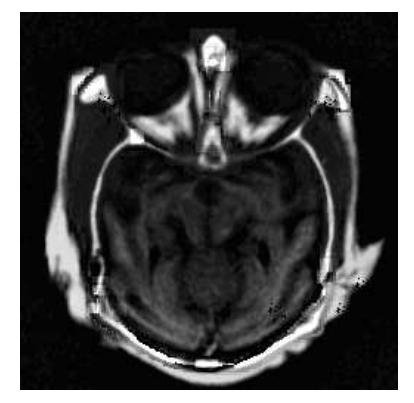

(c) Laplacian pyramid method

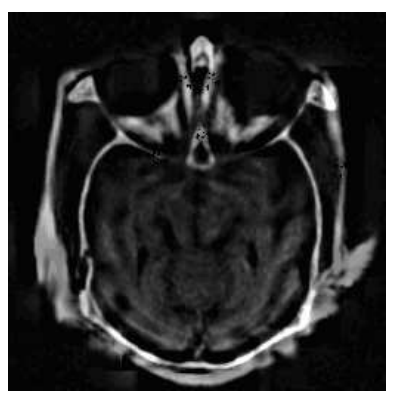

(d) Wavelet transform method

Fig. 1 Source images and fused images.

Except for the visual comparison, two objective indexes, information entropy and average gradient are adopted to perform quantitative analysis for the quality of the fusion image. The magnitude of the value of information entropy reflects the magnitude of information in the fusion image. The average gradient reflects the clarity of the image. The results of specific evaluation are as shown in table1. From table 1 it is known, the fusion effect of the proposed fusion method is good, its image clarity and the information quantity are improved greatly.

Table 1. Objective Evaluation of Three Fusion Algorithms

\begin{tabular}{ccc}
\hline Algorithms & $\begin{array}{c}\text { Information } \\
\text { Entropy }\end{array}$ & Average Gradient \\
\hline PCA & 9.4352 & 12.6789 \\
Wavelet Transform & 10.6789 & 20.2356 \\
Proposed & 14.8463 & 38.3761 \\
\hline
\end{tabular}




\section{Conclusions}

For reasons that the wavelet transform is possessed of good frequency property; morphology is possessed of the merits of smooth extracting edge, not sensitive for noise and less operation quantity; improved cross entropy is possessed of the ability to measure the difference between two images. A method in combination with wavelet transform, morphology and improved cross entropy is adopted, an image fusion algorithm, which is favorable to remain edges, is proposed. This makes the fused image with clear edge, rich details and good visual effect, and offers a good foundation for the follow-up images in property extracting, classification, target identification and so on.

\section{References}

[1] B. Liu, Q. Zhu, J. X. Deng, Fusion Method of Multispectral Image Based on Red-Black Wavelet Transform, Chinese Journal of Scientific Instrument. 32 (2011) 408-414.

[2] H. Wu, H. S. Wang, Sobel Operator and Wavelet Transform, Computer Simulation. 28 (2011) $232-235$.

[3] K. Amolins, Y. Zhang, P. Dare, Wavelet-based Image Fusion Techniques: An Introduction, Review and Comparion, Photogrammetry \& Remote Sensing. 62 (2007) 249-263.

[4] Z. G. Wu, Y. J. Wang, G. J. Li, Application of Adaptive PCNN Based on Wavelet Transform to Image Fusion, Optics and Precision Engineering. 18 (2010) 708-715.

[5] G. Piella, A General Framework for Multiresolution Image Fusion: from Pixels to Regions, Information Fusion. 68 (2003) 259-280.

[6] G. Q. Tao, D. P. Li, G. H., On Image Fusion Based on Different Fusion Rules of Wavelet Transform, Acta Photonica Sinica. 33 (2003) 221-224.

[7] Y. K. Sun, Wavelet Analysis and Application. China Machine Press, first ed., Beijing, 2005.

[8] S. C. Pei, F. C. Chen, Hierarchical Image Representation by Mathematical Morphology Subband Decomposition, Pattern Recognition Letters. 16 (1995) 183-192.

[9] L. Zhou, Z. Y. Wang et al. A New Wavelet Image Fusion Algorithm Based on Human Visual System, Journal of Image and Graphics. 9 (2004) 1088-1094.

[10]L. P. Yan, B. S. Liu, D. H. Zhou, Novel Image Fusion Algorithm with Novel Performance Evaluation Method, Systems Engineering and Electronics. 29 (2007) 509-513. 